\title{
Primary particles of cosmic rays energy determination by measurements of Cherenkov and radio emissions of air showers
}

\author{
Igor Petrov* \\ Yu.G. Shafer Institute of Cosmophysical Research and Aeronomy \\ E-mail: igor.petrov@ikfia.ysn.ru

\section{Stanislav Knurenko} \\ Yu.G. Shafer Institute of Cosmophysical Research and Aeronomy \\ E-mail: knurenkodikfia.ysn.ru

\section{Zim Petrov} \\ Yu.G. Shafer Institute of Cosmophysical Research and Aeronomy \\ E-mail: pzedikfia.ysn.ru
}

\begin{abstract}
The paper considers two independent methods of primary particles determination of cosmic origin. The particles give a rise to nuclear processes that generate cascade multiplication of various types of elementary particles. Methods are based on Cherenkov light registration at optical range of wavelength, formed by the interaction of secondary particles with the atmosphere and transition of charged particles through the Earth's magnetic field with the generation of radio emission. The paper presents empirical formulas based on measurements of air shower characteristics at the sea level. Cross-correlation of air shower energy estimation obtained independently are considered.
\end{abstract}

35th International Cosmic Ray Conference - ICRC2017-

12-20 July, 2017

Bexco, Busan, Korea

\footnotetext{
* Speaker.
} 


\section{Introduction}

The study of cosmic rays (CR) of ultrahigh energies first of all requires an estimate of the energy, the spectrum in terms of energies, masses, and the anisotropy of the arrival of primary particles. This can only be done by calculating the energies of all the secondary particles formed during the interaction of the primary particle with the nuclei of the air atoms [1]. First of all, this is the registration of Cherenkov and ionization radiation [2,3]. According to model calculations, the loss of ionization of air by particles is spent up to $\sim 80 \%$ of the total energy of the primary particle [4]. The rest of the energy is spent on nuclear interactions of hadrons (nucleons) and is carried away by high-energy muons at sea level. In the hybrid registration of air showers, i.e. electron, muon, and Cherenkov components, we can empirically estimate the energy of the primary CR particle. Such a method has been developed and is being used to this day at the Yakutsk complex installation of the EAS. A detailed description of this method is given in [5, 6]. As an alternative to the energy balance method, we describe below the method of independent estimation of E0 obtained by measuring the radio emission of EAS at the frequency of $30-35 \mathrm{MHz}$ at the Yakutsk array.

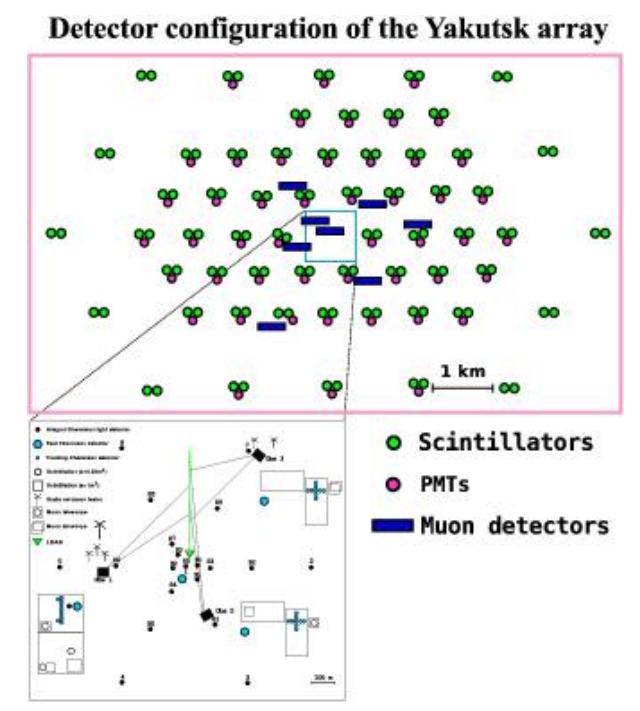

Figure 1: Arrangement on observation stations at the Small Cherenkov array.

The time resolution of the coincidence circuit is $2 \mu \mathrm{s}$. In this case, the signals from the detectors are converted into a digital code and stored in the buffer memory. At the same time, a trigger signal is sent via the communication line to the central recording device for selection of showers.

Selection of air shower events is carried out with the simultaneous triggering of three neighboring stations forming a triangle. The time resolution of the coincidence triangle is $40 \mu \mathrm{s}$. After receiving trigger signal all stations and detectors are sending their data to the central station. Information from the stations and commands from the center to the station are transmitted via the fiber optic link.

In addition to the listed methods for registering various shower components at the Yakutsk EAS array, since 2009, measurements of radio emission from showers with $\mathrm{E}_{0}>10^{17} \mathrm{eV}$ have 
been carried out.

\section{Air shower energy estimation}

\subsection{Energy balance method}

The method of determining the energy at the Yakutsk array is based on the expression (2.1) used in $[1,2]$ :

$$
E_{0}=E_{e i}+E_{e p h}+E_{\mu v}+E_{d}(1)
$$

In the first approximation, the sum of the components presented in (2.1) will be the total energy of the primary particle. All summand of $\mathrm{E}_{0}$ were reconstructed with use of integral parameters of air shower. In our case, they are determined by the following method. The energy, scattered in the atmosphere above observation level by electrons is determined by formula (2.2):

$$
E_{e i}=k\left(x, P_{\lambda}\right) \cdot F
$$

Here $\mathrm{F}$ - total flux of Cherenkov light of air shower; $\mathrm{k}\left(\mathrm{x}, \mathrm{P}_{\lambda}\right)$ - fitting coefficient that takes into account the transparency of the real atmosphere and expressed through the depth of the development of the EAS measured at the array [5]:

$$
E_{e p h}=2.2 \cdot 10^{6} N_{s}\left(X_{0}\right) \cdot \lambda_{e f f}
$$

where $\mathrm{N}_{s}\left(X_{0}\right)$ - total number of charged particles at the sea level, $\lambda_{e f f^{-}}$mean free path of air shower particles, which was found from correlation parameteres $\mathrm{N}_{s}-\mathrm{Q}(400)$ at different zenith angles [7]. The energy transferred into muons with threshold energy $\geq 1 \mathrm{GeV}$, estimated by the formula (4):

$$
\mu=\varepsilon_{\mu} \cdot N_{\mu}
$$

Where $\varepsilon_{\mu}$ - average muon energy, calculated according to the measured energy spectrum of air shower muons up to $\mathrm{E}_{\mu}=10^{3} \mathrm{GeV}$ and equal to $10.6 \mathrm{GeV}$. $\mathrm{N}_{\mu}$ - total number of muons with threshold energy $\mathrm{E}_{t h r} \geq 1 \mathrm{GeV}$.

Remaining insignificant part ( $\sim 5 \%)$ of primary energy that is difficult to estimate experimentally we determined from calculations. Magnitude of ionization losses of muons is equal to $\mathrm{E}_{\mu i}$ $=(0.12 \pm 0.09) \mathrm{E}_{\mu}$, energy losses on nuclear fission in the air as $0.5 \mathrm{GeV}$, ionization losses of hadron component in the atmosphere as $\mathrm{E}_{h i}=(5.6 \pm 2.2) \cdot 10^{-2} \cdot E_{e i}$ and the energy corresponding for neutrino component as $\mathrm{E}_{v}=(0.64 \pm 0.18) \cdot E_{\mu}$.

Uncertainty of the method for estimating total energy of air shower in our case is $25 \%$. Further this energy estimation is used to establish a relation of the radio emission amplitude with EAS energy [8].

\subsection{Radio emission amplitude correlation with air shower energy}

Fig. 2 and 3 show data obtained from measurements of radio emission at the Yakutsk array. On the OY axis - the number of showers n, along the axis OX - the energy of showers. In Fig. 2 along the OY axis - the number of showers n, along the axis OY - cos of the zenith angle $\theta$. 


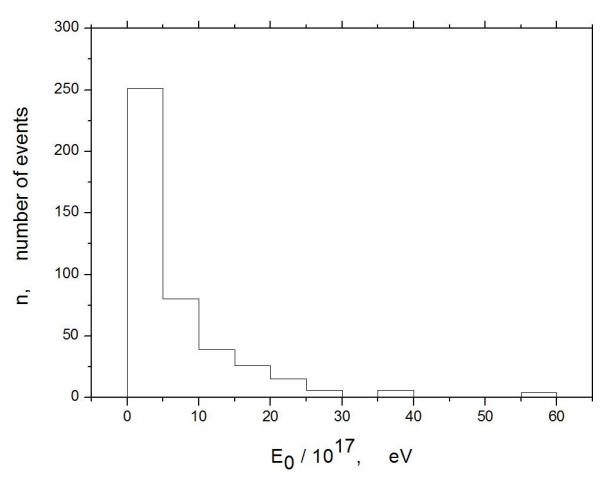

Figure 2: Distribution of showers by energy.

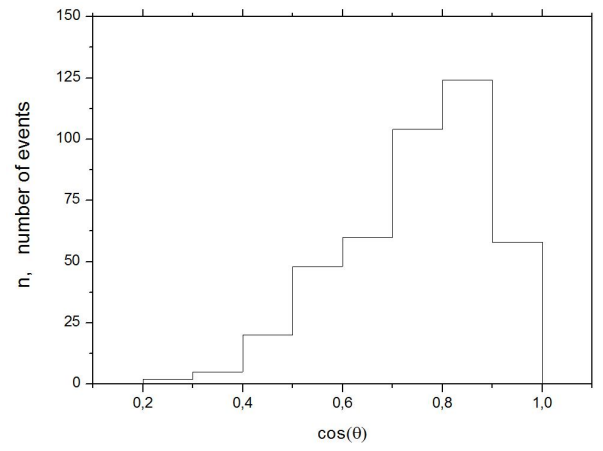

Figure 3: Distribution of showers at the zenith angle $(\cos \theta)$.

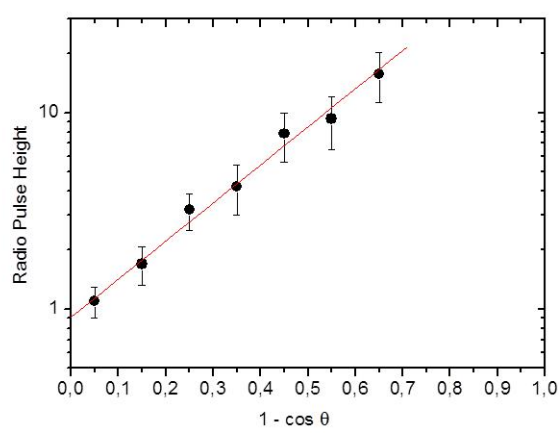

Figure 4: Dependence of the amplitude of radio emission from the zenith angle.

In Fig. 4 shows the dependence of the amplitude of the radio emission from the zenith angle. The dependence obtained is described by the formula:

$$
\varepsilon_{E W}=(0.81 \pm 0.25)(1-\cos \theta)^{1.16 \pm 0.05}
$$

Fig. 5 shows distribution of the distances of antennas from showers axis. In Fig. 5 shown that, the majority of antennas are in the range of $200-500 \mathrm{~m}$. For this reason, median average distance $\left.<R_{\text {med }}\right\rangle=350 \mathrm{~m}$ was taken for normalization. Amplitude of antennas at different distances from shower axis were normalized to $\left\langle R_{\text {med }}>\right.$ according to eq. (2.1). According to the same data, the 


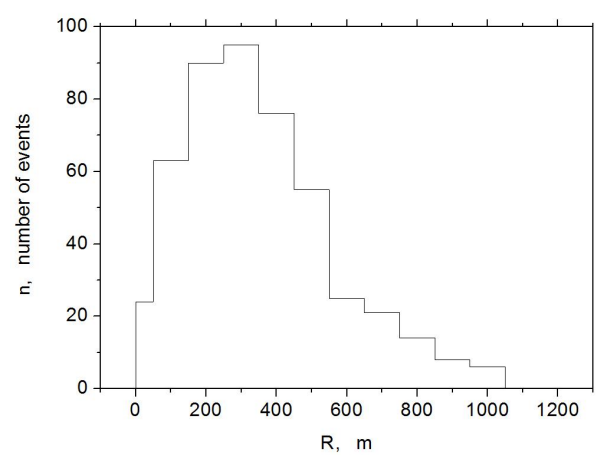

Figure 5: Distribution of antenna distances from air shower axis

dependence of the amplitude of the radio signal on the cos of the zenith angle was also found [10]. The energy of the shower was determined from the total flux of Cherenkov light from the EAS (see 2.1) [2].

The radio signals thus normalized were involved in plotting the dependence of the magnitude of the signal on the shower energy (Fig. 6).

$$
\varepsilon_{E W}=(1.3 \pm 0.3)\left(\frac{E_{0}}{10^{17}} e V\right)^{0.99 \pm 0.04}
$$

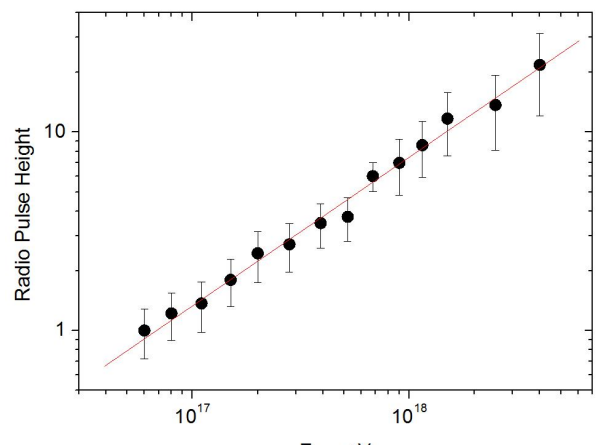

Figure 6: Dependence of radio emission amplitude on air shower energy

Electric field intensity at radio array antennas, according to [11], can be shown as:

$$
\varepsilon_{v}=\sqrt{\frac{4 \pi v^{2} \mu_{0}}{G_{\theta, \varphi, v}} \frac{1}{K_{\text {ele }}(v) R_{A D C}} \cdot V_{A D C}}
$$

where $v$ - observation frequency $30-35 \mathrm{MHz}, \mathrm{G}(\theta, \varphi)$ - antenna gain, depends on direction, $K_{\text {ele }(v)}$ - full gain of amplification electronics, $\mathrm{V}_{A D C}-\mathrm{ADC}$ value and $\mathrm{R}_{A D C}$ - $\mathrm{ADC}$ resistance $\mathrm{R}_{A D C}$ $=50 \Omega$.

It also shows that radio pulse amplitude $\mathrm{A}_{\max }$ is proportional to the energy of the electromagnetic component of air shower:

$$
E_{E M}=c \cdot A_{\max }
$$


where $\mathrm{c}$ - parameter, that characterizes power of the shower.

The amplitude of the radio emission signal can be measured by an antenna with an east-west direction and can be represented as a function that depends on the zenith angle, the distance from the shower axis and the energy of the primary particle, which was determined from the data of the Yakutsk array.

$$
\begin{array}{r}
\varepsilon(E, \theta, R)=(15 \pm 1)(1-\cos \theta)^{1.16 \pm 0.05} \\
\exp \left(-\frac{R}{350 \pm 25.41}\right) \cdot\left(E_{0} / 10^{17} e V\right)^{0.99 \pm 0.04}
\end{array}
$$

\section{Conclusion}

With the use of antenna parameters and hardware properties (pre amplifier and post amplifiers) at the Yakutsk array [12], we derived air shower energy estimation by radio emission data [9].

By measuring electric field with radio antennas it is possible independently estimate air shower energy and compare two estimations. The differences between two estimations would characterize precision of air shower energy estimation.

\section{Acknowledgments}

The reported study was funded by RFBR according to the research project 16-29-13019.

\section{References}

[1] M.N. Dyakonov, S.P. Knurenko, V.A. Kolosov et al. Nuclear Instruments and Meth-s in Phys. Rec. Amst., A248, 4, 224-226 (1986)

[2] S.P. Knurenko, A.A. Ivanov, I.Ye. Sleptsov, A.V. Sabourov. JETP Letters, 3, 11, 563 (2006)

[3] C. Song, Z. Cao, B.R. Dawson et al. // Astroparticle Physics. 14, 7-13, (2000).

[4] A.A. Belyaev, I.P. Ivanenko, B.L. Kanevsky et al. - M.: Science, 305 (1980)

[5] A.A. Ivanov, S.P. Knurenko, I.Ye. Sleptsov // New Journal of Physics. 11, 065008 (2009)

[6] A.V. Glushkov, M.N. Dyakonov, T.A. Egorov et al. Bulletin of RAS, 57, 91-93 (1993)

[7] A.A. Ivanov, S.P. Knurenko, I.Ye. Sleptsov. JETP, 131, 1001, 6 (2007)

[8] S.P. Knurenko and I.S. Petrov. Bulletin of RAS. Physics series. 79, 3, 446-448 (2015)

[9] S. Knurenko, V. Kozlov, Z. Petrov, M. Pravdin, Bulletin of Russian Academy of Sciences. Physics 77, 1559-1560 (2013)

[10] A. Horneffer et al. // Proc. 30th ICRC. Merida. 4, 83 (2007)

[11] S.P. Knurenko, D.S. Borschevsky, Z.E. Petrov, I.S. Petrov. Proc. SPIE 8696, 86960Q (2012). 